\title{
Lung Ultrasound in Corona Pandemics: \\ How Can Lung US Assist Early Diagnosis of COVID-19 \\ Pneumonia?
}

\author{
Enas Khalifa Ahmed Abuzied ${ }^{1}$, Ashraf Zein El Abidien Mohamed ${ }^{2}$, \\ Khaled Fawzy Alkhayat ${ }^{1}$, Hisham A. Alghany Algahlan ${ }^{3}$, Hamdy A. \\ Mohammadien ${ }^{1}$
}

${ }^{1}$ Department of Chest diseases and Tuberculosis, Faculty of medicine, Sohag University

${ }^{2}$ Department of Chest diseases and Tuberculosis, Faculty of medicine, Assuit University

${ }^{3}$ Department of Radiology, Faculty of medicine, Sohag University.

\begin{abstract}
:
Up-to-date review of the role of lung ultrasound (US) in early diagnosis of COVID-19 disease associated pneumonia, based on the recent publications at the 2019/2020 pandemic of nCoV-19. Further, highlighting the advantages of lung US over the other imaging modalities as regard early diagnosis, cost and potency of its use as a screening tool in pandemics. The COVID-19 tends to be bilateral with patchy pattern. More frequent in the posterior and inferior fields of the lung. This infiltration is usually irregular and extends to the pleural line. Diffuse B-lines are noticed with air bronchogram sign. Sometimes localized pleural thickening is seen or localized pleural effusion. Occasionally, impaired blood flow in the consolidation patch was detected.

In novel COVID-19 era lung US is a bedside, practical, safe and useful emergency tool for diagnosis. It is operator dependent and this drawback can be avoided by providing proper training.
\end{abstract}

Keyword: Lung ultrasonography, COVID-19 Pneumonia and Corona virus.

\section{Introduction}

Since the beginning in December 2019, a pool of cases with pneumonia of unknown and unexplained etiology was registered in Wuhan, Hubei province in China (1). On Jan 7, 2020, the diagnosis of novel coronavirus, severe acute respiratory syndrome coronavirus2 (SARS-CoV-2) was recognized as the responsible organism by Chinese authorities. They performed deep sequencing analysis of nasopharyngeal swabs. By the end of January of 2020, the WHO confirmed the outbreak of the disease and it was considered as a global health emergency (2).

The first 41 patients who confirmed to have SARS CoV-2 infection presented with lower respiratory tract infection with fever, dry cough, and dyspnea. Those manifestations are similar to the two other diseases caused by coronaviruses; severe acute respiratory syndrome (SARS) and Middle East respiratory syndrome(MERS) (3).

The distinctive features which raised the suspicion towards the diagnosis of the new disease are the ground-glass pattern of pneumonia in chest computed tomography (CT) and lymphocytopenia (in $83.2 \%$ of the patients on admission) (4).

The diagnosis was made based upon the WHO criteria published on Jan 12, 2020. Later, all the cases were confirmed by real-time RT-PCR analysis of 
nasopharyngeal swab (5). It was agreed that lung CT is strongly recommended in suspected cases, as the respiratory system is primarily involved in the disease. Moreover, it can be used for follow-up (6).

Noteworthy, lung involvement may precede clinical manifestations and even PCR +Ve swab. Accordingly, experts recommend early chest $\mathrm{CT}$ for screening suspected patients (7). Likewise, a current study revealed that the CT chest is more sensitive than real time-PCR in discovering $\mathrm{nCoV}-19$ infection. This can be attributed to defect in nucleic acid detection technology, low viral load or inappropriate clinical sampling (8).

Feng et al, reported that chest CT has a crucial role in the diagnosis and appraisal of lung affection and damage in COVID-19 disease (9).

The extraordinary contagiousness of SARSCoV-2 and the hazards of moving patients who is hypoxemic and with hemodynamic instability, hamper the use of chest CT on a wide scale especially in pandemics. Further, radiation hazards, long examination time and its unavailability in primary healthcare centers all are obstacles limiting its use and necessitate the use of more practical alternative (10).

Lung US is a highly sensitive and specific approach that can be used instead of CT scanning, it provides comparable quality to $\mathrm{CT}$ and it is outweighing standard radiography for evaluation of pneumonia and adult respiratory distress syndrome (ARDS) with the additional value of being used easily and can be repeated without exposure to radiation hazards (11).

-COVID-19 Pneumonia is different from the previously known pneumonias transmitted by influenza virus in being rapidly transmitted and in having high infection and mortality rate. It can be transmitted by droplets and direct contact; so the device of ultrasonic waves can be easier disinfected than CT and CXR due to its smaller area of contact with patients. (12)

\section{Lung Ultasonograpic manifestations in patients with COVID-19 disease}

-In all studies, the thorax was scanned in the following 12 lung areas at bedside: anterior superior and inferior, lateral superior and inferior, posterior superior and inferior, bilaterally using " The 12-area examination method" (13).

-The lesions(COVID-19 foci) tend to be bilateral with patchy pattern. More frequent in the posterior and inferior fields of the lung. This infiltration is usually irregular and extends to the pleural line. Diffuse B-lines are noticed with air bronchogram sign as shown in Figure (1) and (2). Sometimes localized pleural thickening and pleural effuseeion are observed .

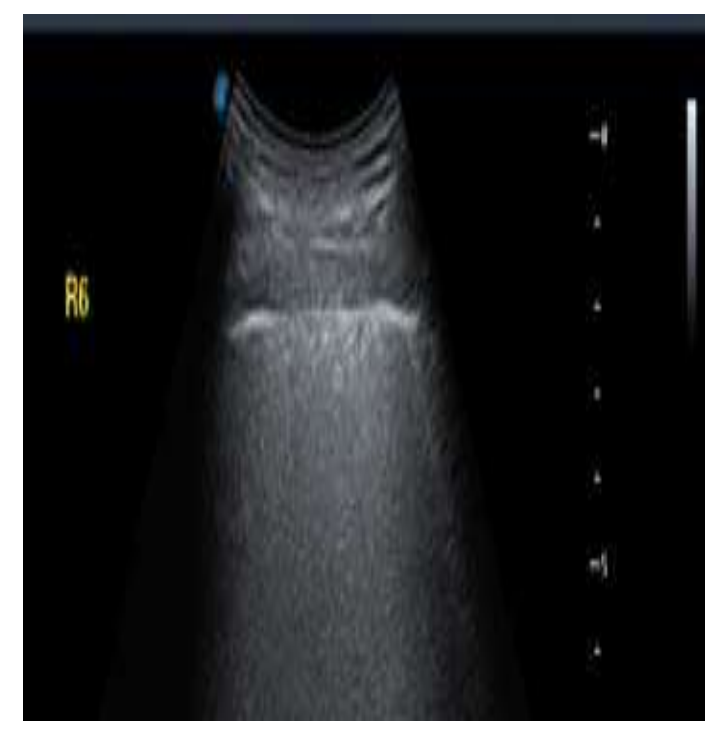

Figure(1): the convex array probe showed the pleural line in the right posterior

lower area was unsmooth and thin with diffused B lines and"white lung" sign (12). 
SOHAG MEDICAL JOURNAL

Vol. 24 No. 2 April 2020

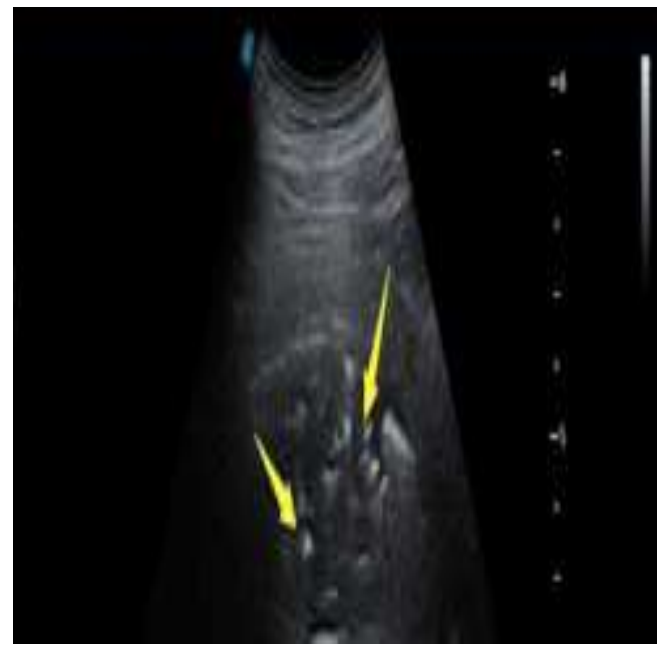

Figure(2) :convex array probe showed large areas of consolidation in the right posterior upper area and airbronchogram sign (yellow arrow). The pleural line was interrupted (12).

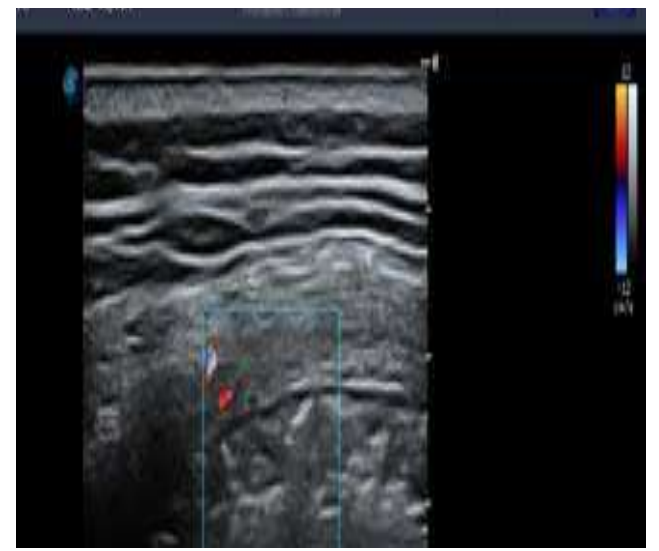

Figure(3): color Doppler ultrasound showed no obvious blood flow signal in the peri-pulmonary consolidation of the left posterior upper area, which was significantly different from that of common inflammatory bacterial pneumonia (12)

-Yi Huang et al, reported that the noncritical COVID-19 has specific ultrasonographic findings, which are observed in the posterior and inferior fields of the lung in the form of multiple B lines, sub-pleural pulmonary consolidation and poor blood flow. He used SonoScape P50 color ultrasonic device, with frequency of convex array probe was $1-8 \mathrm{MHz}$ and that of linear array probe was 3-17 $\mathrm{MHz}$ (12).

-In another study performed on 20 cases with COVID-19 disease, QianYi Peng et al found the following characteristic features:

1) pleural line is thickened and irregular.

2) Focal or diffuse B lines.

3) Consolidations with mostly dynamic air bronchogram.

4) Pleural effusions are rare.

5) A lines appear in the convalescence.

-These noticed features occurred through different stages of the disease, from early stage of alveolar interstitial pattern, to the more advanced stage of pulmonary consolidation and bilateral interstitial pattern (14).

-In a third study, Erika Poggiali et al found that there was strong correlation between bedside US findings and Chest CT scans performed for 12 patients who presented with flu-like symptoms and COVID-19 infection during the previous 4-10 days, diffuse $B$ lines with spared areas was found in all patients, subpleural consolidation was found only in three patients (15). -In fourth study, D. BUONSENSO et al reported a case of COVID-19 disease whose Lung ultrasound showed, an irregular pleural line with small subpleural consolidations, areas of white lung and thick, confluents and irregular vertical artifacts(B-lines) on the anterior and posterior fields bilaterally.(16)

-Color Doppler technique is able to show the blood supply of the lesion, which has a strong clinical signifycance, poor blood flow signal is usually observed in the sub-pleural consoledation, this may be due to the rapid progression of COVID-19, therefore the microvessel exchange mechanism is not quickly established by the lung Figure(3),(17). 
Lung Ultrasonic findings Versus

\section{Chest CT findings}

-Bedside US findings showed strong correlation with Chest CT scans in the different stages of the disease, Qian-Yi Peng et al described a comparison between lung ultrasonographic manifestations and chest CT findings in patients with COVID-19 disease as the following (14).

* In the early stage of disease: Lung US show Focal B lines while Lung CT may be negative or atypical.

* In the progressive stage of disease: Lung US show alveolar interstitial syndrome correspondding to the fuse or scatterd ground glass opacities seen in Lung CT.

- Further, lung consolidation is seen in the form of airbronchogram in Lung US and usually subpleural and translobar in Lung CT.

- In case of recovery: A lines can be found while uneven B lines can be seen in patients developed pulmonary fibrosis in the Lung US.

* In both US and CT: there is pleural thickening while pleural effusion is mostly rare .

\section{Advantages of Lung US over other imaging modalities:}

1-Lung images can be obtained at bedside by the same evaluating clinician, so we success in reducing the number of health workers potentially exposed to the patient. Nowadays, the use of traditional imaging such as Chest XRay or CT Chest require movement of the patients to the radiology unit and potentially many people can be exposed, from health care professional to the other patients waiting imaging for other diseases.

2- Lung ultrasound can help in first screening and discriminating low-risk patients (those with lung ultrasoundnegative patients) from higher-risk patients (abnormal lung ultrasound patterns), who urgently require second level imaging or even experimental therapies without waiting.

3- Lung ultrasound would be much easier to use as a screening tool than chest $\mathrm{CT}$, and this doesn't decrease the role of chest $\mathrm{CT}$, but routine use of $\mathrm{CT}$ scan has several obvious limitations.

4- Lung ultrasound is easier to be sterilized than chest CT especially if portable due to the smaller surface area.

5-Lung ultrasound avoid radiation exposure so: -

a) It can be repeated several times for the same patient which allow a close monitoring of the clinical condition

b) It allows imaging of pregnant patients and patients with renal impairment who Chest CT may harm them.

6- Lung ultrasound can be easily performed in the outpatient setting allowing better pre-triage evaluation to determine those patients that should be sent to the hospital.

7- Lung ultrasound is a cheap instrument that can be easily applied even in poor settings.

8-Lung ultrasound produce real-time images, it is more sensitive than $\mathrm{CT}$ in detection of pleural effusion and interstitial lesions, it is also characterized by showing the blood flow distribution in the inflammatory lesions which is an indicator of the degree of angiogenesis and progression of the disease. - Lung ultrasonography has a known limitation as it cannot detect central lesions that are deep within the lung, as air inside the lung acts as a barrier against transmission of ultrasonic waves, fortunatey this is uncommon in COVID-19 disease, where most lesions located peripherally and sub-pleural.

\section{Conclusion}

Lung US is useful as a screening tool for diagnosis of COVID-19 pneumonia 
in emergency department. It reduces the infection probability of health workers, can be repeated, no radiation and it can be applied on wide base in pandemics. Posterior and inferior lung infiltration with large number of Blines are distinguishing Lung US features for COVID-19 pneumonia. Sub-pleural consolidation with impairred blood flow are additional features.

\section{References:}

1. Zhu N, Zhang D, Wang W, Li X, Yang B, Song J, et al. China Novel Coronavirus Investigating and Research Team. A novel coronavirus from patients with pneumonia in China, 2019. N Engl J Med. 2020;382(8):727-33.

2. Novel CPERE. The epidemiological characteristics of an outbreak of 2019 novel coronavirus diseases (COVID19) in China. Zhonghua liu xing bing xue za zhi $=$ Zhonghua liuxingbingxue zazhi. 2020;41(2):145.

3. Huang $\mathrm{C}$, Wang $\mathrm{Y}$, Li $\mathrm{X}$, Ren $\mathrm{L}$, Zhao J, Hu Y, et al. Clinical features of patients infectedwith 2019 novel coronavirus in Wuhan, China. The Lancet. 2020;395(10223):497-506.

4. Guan W-j, Ni Z-y, Hu Y, Liang W-h, $\mathrm{Ou}$ C-q, He J-x, et al. Clinical characteristics of coronavirus disease 2019 in China. New England Journal of Medicine. 2020.

5. Organization WH. Clinical management of severe acute respiratory infection when novel coronavirus $(\mathrm{nCoV})$ infection is suspected: interim guidance, 25 January 2020. World Health Organization, 2020.

6. Jin Y-H, Cai L, Cheng Z-S, Cheng H, Deng T, Fan Y-P, etal. A rapid advice guideline for the diagnosis and treatment of 2019 novel coronavirus (2019-nCoV) infected pneumonia (standard version). Military Medical Research. 2020;7(1):4.

7. Jin YH, Cai L, Cheng ZS, Cheng $\mathrm{H}$, Deng T, Fan YP, et al. A rapid advice guideline for the diagnosis and treatment of 2019 novel coronavirus (2019-nCoV) infected pneumonia (standard version). Mil Med Res. 2020;7(1):4.

8. Fang Y, Zhang H, Xie J, Lin M, Ying L, Pang P, et al. Sensitivity of Chest CT for COVID-19: Comparison to RT-PCR. Radiology. 2020:200432.

9. Pan F, Ye T, Sun P, Gui S, Liang B, Li L, et al. Time Course of Lung Changes On Chest CT During Recovery From 2019 Novel Coronavirus (COVID-19) Pneumonia. Radiology. 2020:200370.

10. Mayo P, Copetti R, Feller-Kopman D, Mathis G, Maury E, Mongodi S, et al. Thoracic ultrasonography: a narrative review. Intensive care medicine. 2019:1-12.

11. Zhou B, Bartholmai BJ, Kalra S, Osborn TG, Zhang X. Lung US Surface Wave Elastography in Interstitial Lung Disease Staging. Radiology.84-479:(2)291؛2019 .

12. Huang Y, Wang S, Liu Y, Zhang Y, Zheng $\mathrm{C}$, Zheng $\mathrm{Y}$, et al. A Preliminary Study on the Ultrasonic Manifestations of Peripulmonary Lesions of Non-Critical Novel Coronavirus Pneumonia (COVID19). Available at SSRN 3544750. 2020.

13. Soummer A, Perbet S, Brisson $H$, Arbelot C, Constantin J-M, Lu Q, et al. Ultrasound assessment of lung aeration loss during a successful weaning trial predicts postextubation distress. Critical care medicine. 2012;40(7):2064-72.

14. Peng Q-Y, Wang X-T, Zhang L-N, Group CCCUS. Findings of lung ultrasonography of novel corona virus pneumonia during the 2019-2020 epidemic. Intensive Care Medicine. 2020:1.

15. . Poggiali E, Dacrema A, Bastoni D, Tinelli V, Demichele E, Mateo Ramos $\mathrm{P}$, et al. Can Lung US Help Critical Care Clinicians in the Early 
Diagnosis of Novel Coronavirus (COVID-19) Pneumonia? Radiology. 2020:200847.

16. . Buonsenso D, Piano A, Raffaelli F, Bonadia N, DONATI KDG, Franceschi F. novel coronavirus disease-19 pnemoniae: a case report and potential applications during COVID-19 outbreak. European Review for Medical and
Pharmacological 2020;24:2776-80.

Sciences.

17. . Yin $\mathrm{W}$, Li $Y$, Zeng $X$, Qin $Y$, Wang D, Zou T, et al. The utilization of critical care ultrasound to assess hemodynamics and lung pathology on ICU admission and the potential for predicting outcome. PloS one. 2017;12.(8) 